\title{
Forward calculation of earth-ionosphere model based on quadrature-with-extrapolation algorithm
}

\author{
Guangyun Suo, Diquan LI \\ School of Geosciences and Info-Physics \\ Central South University \\ Changsha 410083,China \\ E-mail ${ }^{1}: 15111208476 @ s i n a . c n$
}

\begin{abstract}
The influence of ionosphere and displacement current can not be ignored in earth-ionosphere mode.This leads to a highly oscillating kernel function and it's hard to get accurate results using traditional fast Hankel transform(FHT) method.This paper attempts to solve this problem using quadrature with extrapolation(QWE) method which Kerry Key proposed in 2012.First,this paper uses analytical solution of CSAMT in uniform half-space to verify the accuracy of this algorithm, then compares calculation results of three-layer model in earth-ionosphere mode with several groups of filter coefficients.It is found that the effect of QWE is much better than FHT.
\end{abstract}

Keywords-earth-ionosphere mode; fast Hankel transform; quadrature with extrapolation

\section{INTRODUCTION}

Extremely low frequency(ELF) electromagnetic method uses strong artificial stationary source to detect underground electrical structure deep within the range of about $10 \mathrm{~km}$. ELF has the characteristics of high signal intensity, strong antiinterference ability and wide coverage.It has great prospects for development in the underground resource exploration and earthquake forecasting.

However, it also brings some new problems. Because of the long-range of ELF, the impact of the ionosphere and the displacement current can not be ignored. At this time,the conventional fast Hankel transform method can not meet the needs.
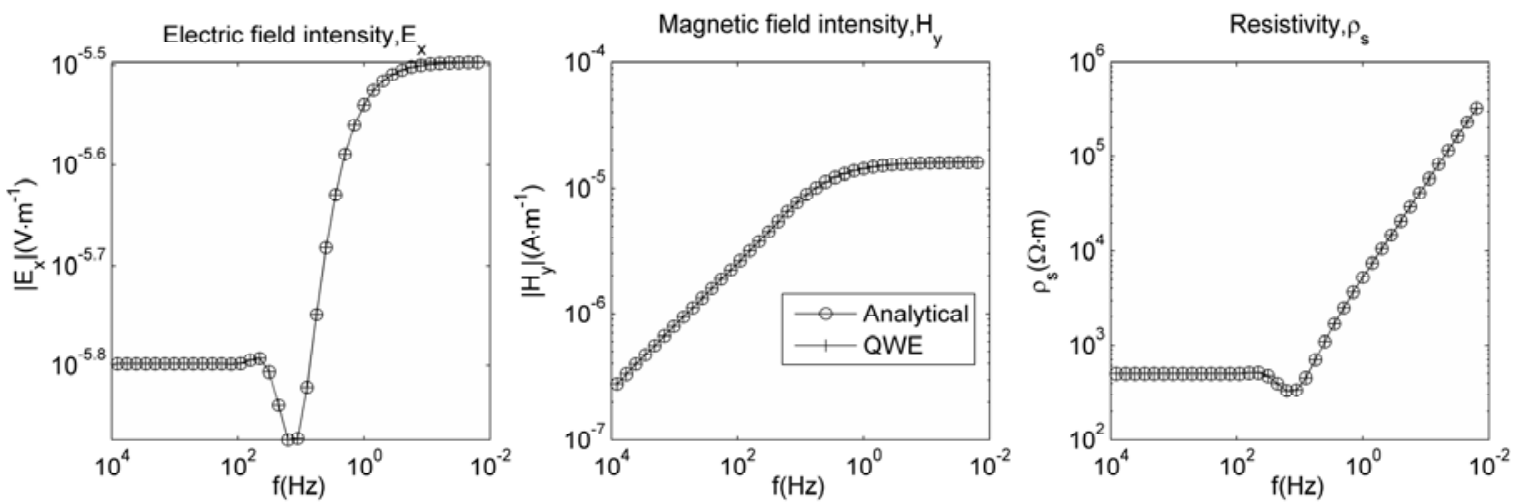

Fig. 1. Analytical and numerical solutions of CSAMT in the uniform half-space.Parameters are as follows: $\sigma=2 \times 10^{-3} \mathrm{~S} / \mathrm{m}, \quad r=10 \mathrm{~km}, \mathrm{I}=10 \mathrm{~A}, \mathrm{AB}=2 \mathrm{~km}, \varphi=0, f=2^{\mathrm{n}} \mathrm{Hz}, \mathrm{n}$ is $-6: 0.5: 13$.
Li Diquan made a very detailed study about forward calculation of earth-ionosphere model in [1]. This article attempts to calculate Hankel transform using quadrature with extrapolation(QWE) algorithm which uses Gauss quadrature to calculate partial integrals in finite intervals and uses the Shanks transformation computed with Wynn' $s \quad \varepsilon$ algorithm to accelerate the sum of partial integrals.

\section{QWE ALGORITHM}

QWE algorithm partitions the Hankel integral into an infinite sum of partial integrals, uses Gauss quadrature to evaluate the partial integrals in every intervals and then makes some modification:

$$
\begin{aligned}
F_{\mathrm{i}} & \approx \sum_{j=1}^{m} f\left(x_{j} / r\right) w_{j} J_{v}\left(x_{j}\right)=\sum_{j=1}^{m} f\left(x_{j} / r\right) \hat{J}_{v}\left(x_{j}\right) \\
& =\sum_{j=1}^{m} f\left(x_{j} / r\right) H_{j}
\end{aligned}
$$

Where $\mathrm{H}_{j}$ is independent of the specific argument $r$ and then can be precomputed, much like the filter weights in fast Hankel transform(FHT).Then,the sum of partial integrals can be accelerated using Shanks transformation: 


$$
S\left(A_{n}\right)=\frac{A_{n+1} A_{n-1}-A_{n}^{2}}{A_{n+1}-2 A_{n}+A_{n-1}}
$$

Typically, $S\left(A_{n}\right)$ converges faster than $A_{n}$.To achieve this transformation,Wynn's $\varepsilon$ algorithm is a good choice.

\section{NUMERICAL EXAMPLES}

In order to verify the validity of QWE algorithm,the analytical and numerical solutions of CSAMT in the uniform half-space have been calculated. Fig. 1 shows that numerical solution and analytical solution is exactly the same, indicating that the accuracy of QWE algorithm is guaranteed.

Then,calculate the frequency response of three-layer model in earth-ionosphere mode using 301-point,801-point and 2040point digital filters whose calculation results are relatively good.

Fig.2 shows that at this time,the result of the most widely used 801-point digital filter is totally wrong. While apparent resistivity result calculated by 301-point digital filter is correct,there are still some problems in the electromagnetic field calculation results of high frequencies.

In order to easily compare the result of 2040-point digital filter and QWE method,amplify high-frequency part, encrypt frequency points and let value of $n$ is from 7 to 10.4, interval is 0.2. Other parameters keep unchanged.

Fig,3 shows that similar to 301-point filter,the apparent resistivity calculated by 2040-point filter is correct,while field value results are oscillations at frequencies above $600 \mathrm{~Hz}$.Only QWE algorithm can achieve satisfactory results both in lowfrequency and high-frequency parts. When drawing the radiation patterns,it is clear to see the much difference between these two methods.

Still using the above model and parameters,draw the radiation patterns at $64 \mathrm{~Hz}$ and $1000 \mathrm{~Hz}$ calculated by $2040-$ point filter and QWE method respectively and compare the radiation pattern at $64 \mathrm{~Hz}$ with published results in [1] to verify the accuracy of these results.Given the limited space, here only gives the relative error of QWE method.
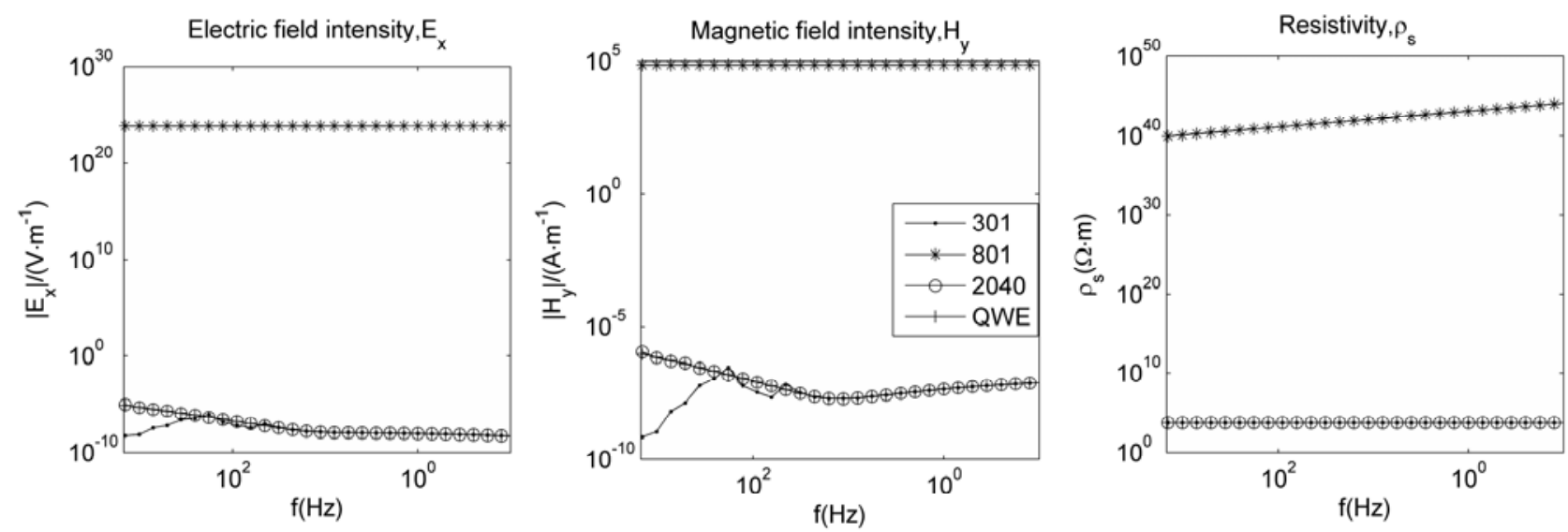

Fig. 2. Frequency response of three-layer model in earth-ionosphere mode using 301-point,801-point and 2040-point digital filters and QWE method.Parameters are as follows: $\sigma=2 \times 10^{-4} \mathrm{~S} / \mathrm{m}, \quad r=2500 \mathrm{~km}, \mathrm{I}=200 \mathrm{~A}, \mathrm{AB}=50 \mathrm{~km}, \varphi=0, f=2^{\mathrm{n}} \mathrm{Hz}, \mathrm{n}$ is $-4: 0.5: 10.5$.
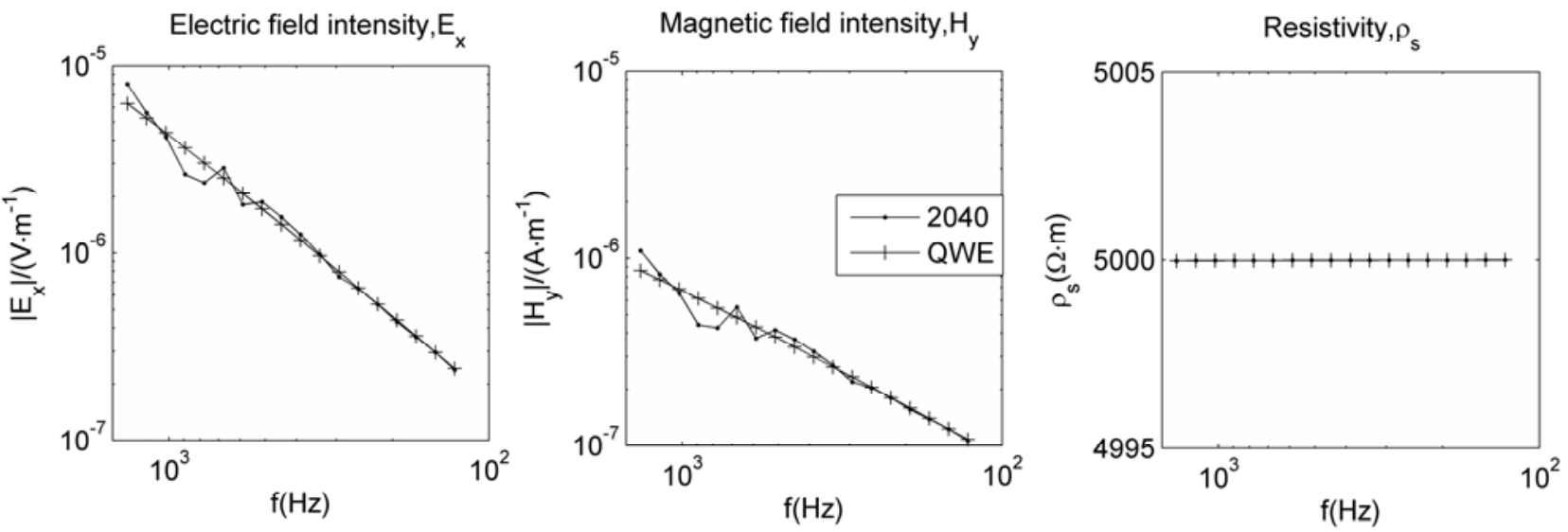

Fig. 3. Frequency response of three-layer model in earth-ionosphere mode using 2040-point filter and QWE method. 

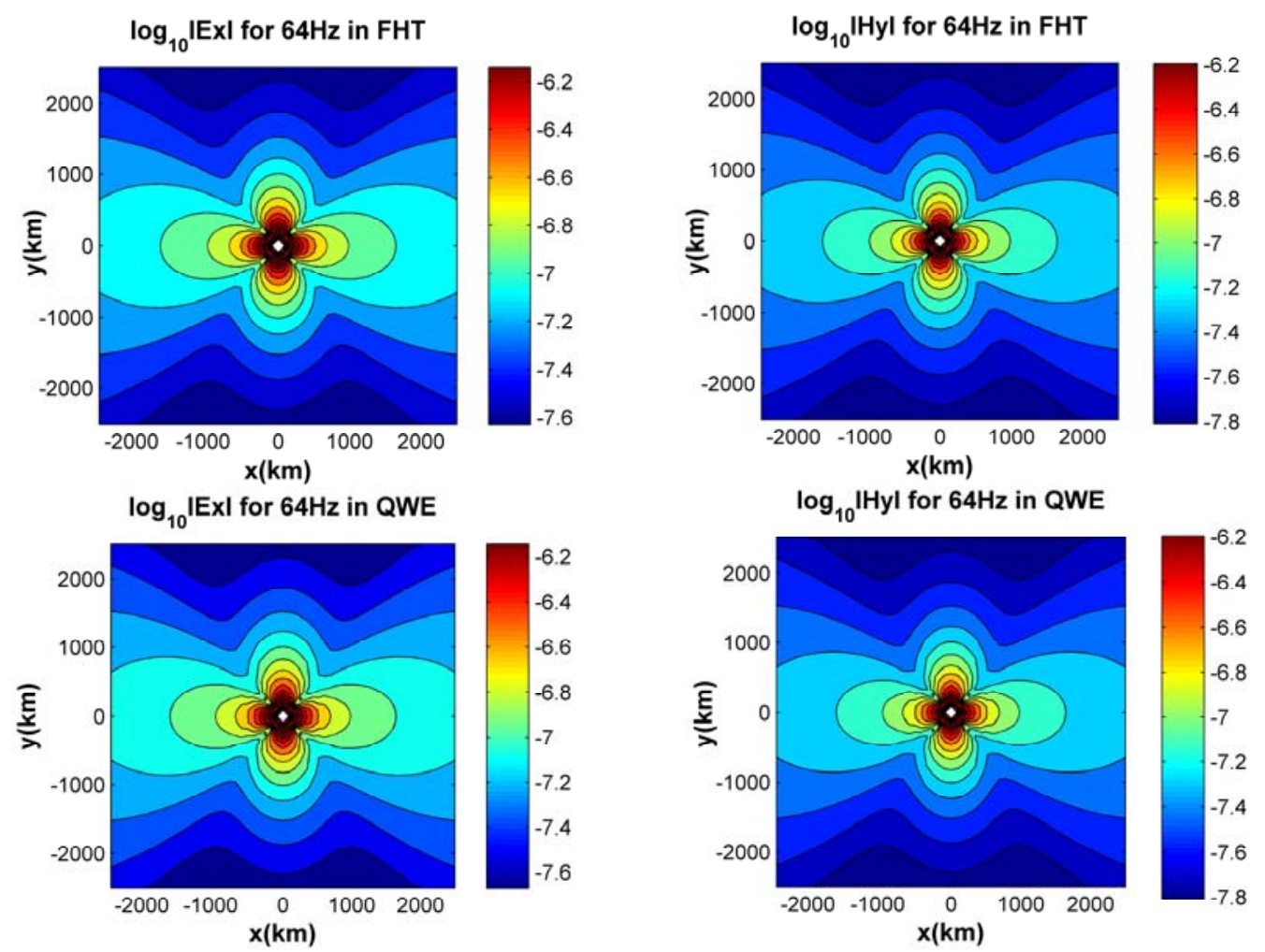

Fig. 4. Radiation pattern at 64Hz.The top and bottom panel shows the result of FHT and QWE methods respectively.

|Ex|

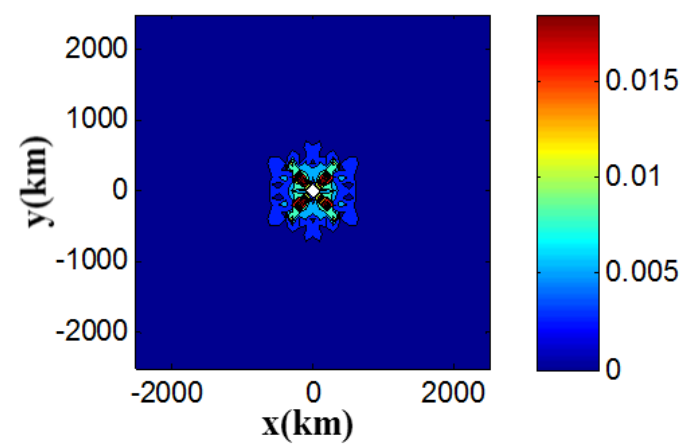

Fig. 5. Relative error of QWE method compared with published results in [1].

Fig. 4 shows that when frequency is $64 \mathrm{~Hz}$, the results using 2040-point filter and QWE method are basically the same and fig. 5 shows that there is only less than $1 \%$ relative error when the offset is at short ranges.It indicates that QWE algorithm is accurate and effective in calculations about earth-ionosphere mode.

When frequency is $1000 \mathrm{~Hz}$,radiation pattern calculated by 2040-point filter coefficients is obviously wrong, or not precise enough.Although it shows a correct change in the overall situation on the value of the electromagnetic field, there are a lot of folds and small closed circles.This point can not be explained in theory, so there must be something wrong in

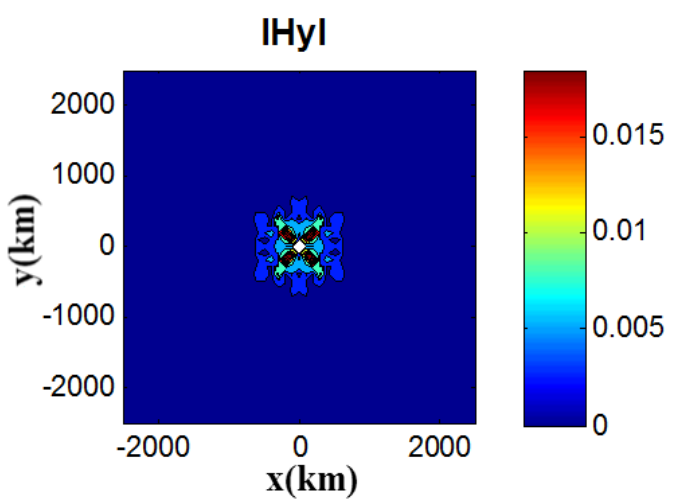

calculation process.But QWE method which uses numerical integration still gets smooth curves.It is because that a group of filter coefficients itself has a cutoff frequency,while numerical integration can adaptively change the order of the points to improve calculation accuracy.

\section{CONCLUSIONS}

As the earth-ionosphere model considers the influence of the ionosphere and displacement current, the traditional FHT algorithm is to fail because of a highly oscillating integral kernel function at high frequencies. However,QWE still can get accurate and effective results. Because of QWE method with high accuracy and stability, it can be easily applied to other 

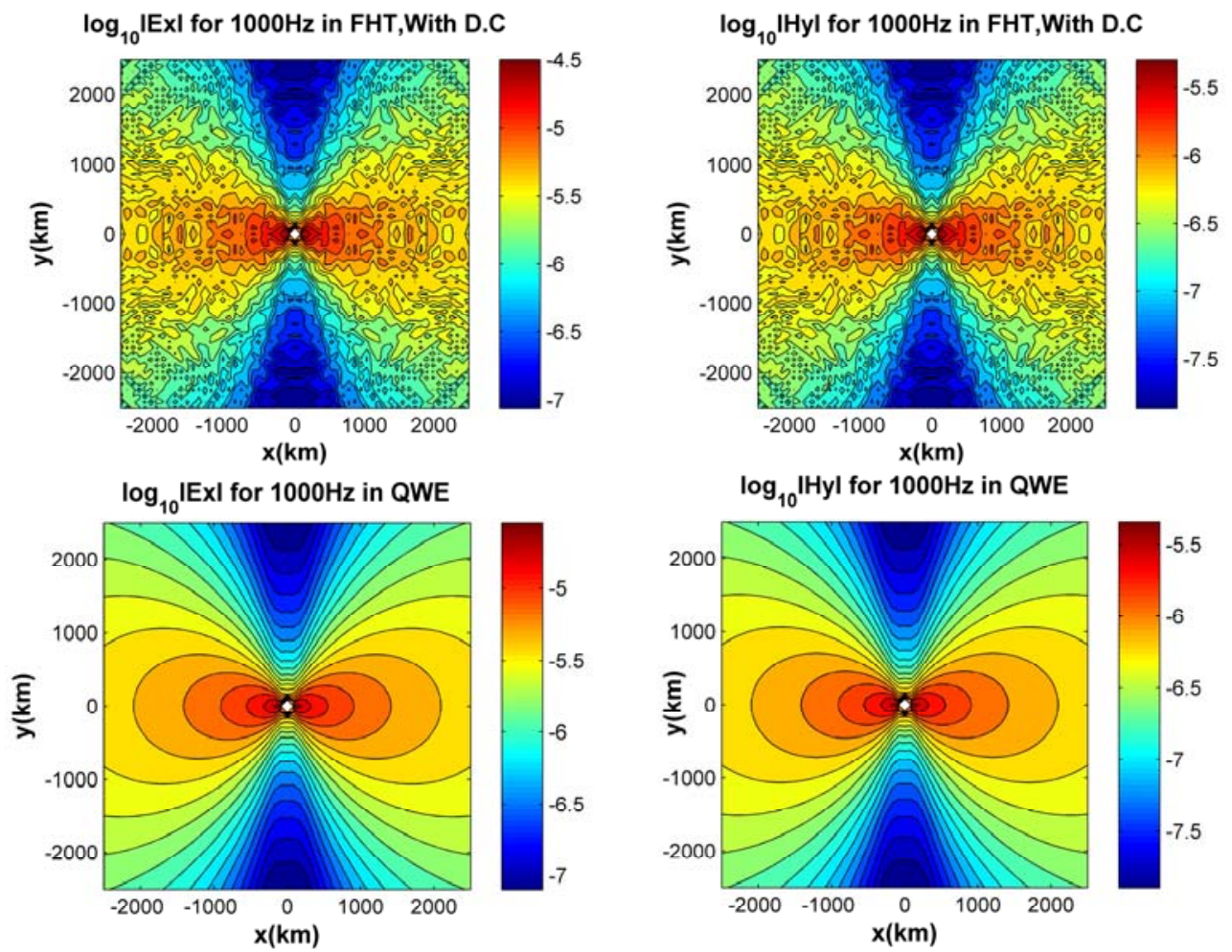

Fig. 6. Radiation pattern at $1000 \mathrm{~Hz}$. The top and bottom panel shows the result of FHT and QWE methods respectively.

geophysical methods relevant to Hankel transform and will also achieve good results.

\section{ACKNOWLEDGMENT}

We would like to thank Dr. Yanfang $\mathrm{Hu}$ and another anonymous reviewers for their constructive advice and comments on our manuscript.

\section{REFERENCES}

[1] K. Kerry, "Is the fast Hankel transform faster than quadrature?" Geophysics, 77,no.3, F21-F30,April 2012. 